\title{
A QUESTION OF KATĚTOV CONCERNING THE HILBERT PARALLELOTOPE
}

\author{
VICTOR KLEE ${ }^{1}$
}

For a linear subspace $L$ of a linear space $E$, it is customary to define the dimensional deficiency of $L$ in $E$ as the algebraic dimension of a linear subspace which is supplementary to $L$ in $E$. The notion is useful even when $E$ is infinite-dimensional. At a recent conference in Warsaw, Professor Katětov asked whether one can define an analogous notion in purely topological terms, at least for the closed subsets of a compact metric space. Such a notion would be useful in applying dimension theory to infinite-dimensional compacta. He suggested the following test-problem: Does the Hilbert parallelotope $P=\left\{x=\left(x^{1}, x^{2}, \cdots\right) \in l^{2}\right.$ : always $\left.x_{n} \in[0,1 / n]\right\}$ admit a self-homeomorphism which carries $A$ onto $B$, where $A=\left\{x \in P: x^{1}=0\right\}$ and $B=\left\{x \in P: x^{1}=x^{2}=0\right\}$ ? Since the sets $A$ and $B$ are intuitively of different dimensional deficiency in $P$, the existence of such a homeomorphism would suggest that the topological notion mentioned above cannot be formulated in a useful way. In the present note, such a homeomorphism is constructed by means of some mapping techniques originated by Keller $[1]$ and developed further by the author $[2 ; 3 ; 4]$. The following more general theorem is proved:

THEOREM. Suppose that (for $\gamma=a, b) K_{\gamma}$ is a compact convex subset of a normed linear space, $H_{\gamma}$ is a proper supporting hyperplane ${ }^{2}$ of $K_{\gamma}$, and $C_{\gamma}$ is an infinite-dimensional closed convex subset of $K_{\gamma} \cap H_{\gamma}$. Then there is a homeomorphism of $K_{a}$ onto $K_{b}$ which carries $C_{a}$ onto $C_{b}$.

The theorem expresses, in particular, a homogeneity property of infinite-dimensional compact convex sets. Other homogeneity properties of such sets have been established by Keller [1] and the author $[2 ; 3 ; 4]$.

A convex subset $S$ of a normed linear space is said to be elliptically convex provided whenever $x$ and $y$ are distinct points of $S$, the open segment ] $x, y$ [ consists entirely of nonsupport points of $S$. Let $Z$ denote the Hilbert space $\left.L^{2}\right] 0, \infty[\times R$, where $R$ is the real number space and $\|(f, r)\|=\left(\|f\|^{2}+r^{2}\right)^{1 / 2}$. For each $r \in R$, let $X_{r}$ denote the 1960.

Presented to the Society January 23, 1961; received by the editors December 13,

${ }^{1}$ Research Fellow of the Alfred P. Sloan Foundation.

2 That is, $K_{\gamma}$ is not contained in $H_{\gamma}$ but is contained in one of the closed halfspaces bounded by $H_{\boldsymbol{\gamma}}$. 
hyperplane $\left.L^{2}\right] 0, \infty\left[\times\{r\} \subset Z\right.$. Let $C^{\prime}$ be an (easily constructed) compact subset of $X_{0}$ which is elliptically convex relative to $X_{0}$, and let $K^{\prime}$ denote the convex hall of $C^{\prime}$ together with the point $(0,1)$. We shall prove the theorem by showing that if $K_{\gamma}$ and $C_{\gamma}$ are as described, then there is a homeomorphism $\zeta_{\gamma}$ of $K_{\gamma}$ onto $K^{\prime}$ which carries $C_{\gamma}$ onto $C^{\prime}$. Then the transformation $\zeta_{b}^{-1} \zeta_{a}$ will be the desired homeomorphism of $K_{a}$ onto $K_{b}$. Since $K_{a}$ and $K_{b}$ enter symmetrically in the hypotheses, we may restrict our attention to a single set $K$, hyperplane $H$, and subset $C$ satisfying the conditions of the theorem. In constructing a homeomorphism $\zeta$ of $K$ onto $K^{\prime}$ such that $\zeta C=C^{\prime}$, we shall rely on the following facts:

I. (See p. 38 of [3].) In a separable normed linear space, a complete convex set which does not lie in any hyperplane must admit a nonsupport point.

II. (See the proofs of IV 1.2 in [2] and 5.4 in [3].) Suppose $B$ is a bounded closed convex subset of a normed linear space $E, 0 \in$ int $B$, and $\mu$ is the gauge functional of $B$. Suppose $S$ is a convex subset of $E$ with $0 \in S$, and $T x=(1+\mu x)^{-1} x$ for each $x \in S$. Then $T$ is a homeomorphism and $T S$ is convex. Suppose further that 0 is a nonsupport point of $S, p_{1}$ and $p_{2}$ are distinct points of $T S$, and $\left.q \in\right] p_{1}, p_{2}[$. If $q$ is a support point of $T S$, then the entire segment $\left[p_{1} / \mu p_{1}, p_{2} / \mu p_{2}\right]$ lies in the boundary of $B$.

III. (See the proof of III 1.1 of [2].) With $Z$ and $X_{0}$ as above, let $\left.\left.Y=L^{2}\right] 0, \infty[\times] 0,1\right] \subset Z$ and let $W$ denote the subspace of $X_{0}$ consisting of all points $(f, 0)$ for which $\left.f \in L^{2}\right] 0, \infty[$ and $f=0$ almost everywhere on $[0,1]$. For each $\left.f \in L^{2}\right] 0, \infty[$ and $t \in[0,1]$, let the function $\left.f_{t} \in L^{2}\right] 0, \infty$ [ be defined as follows:

$$
f_{t} x= \begin{cases}f(x+t-1) & \text { when } x \in[1, \infty], \\ t f(x) & \text { when } x \in] 0,1] .\end{cases}
$$

For each $(f, r) \in Y \cup X_{0}$, let $h(f, r)=\left(f_{r}, r\right)$. Then $h$ is a homeomorphism of $Y \cup X_{0}$ onto $Y \cup W$, each restriction $h \mid X_{r}$ is an affine transformation, $h X_{r}=X_{r}$ for $\left.\left.r \in\right] 0,1\right]$, and $h X_{0}=W$.

Now we are faced with a compact convex subset $K$ of a normed linear space, a proper supporting hyperplane $H$ of $K$, and an infinitedimensional closed convex subset $C$ of $K \cap H$. We wish to construct a homeomorphism $\zeta$ of $K$ onto the set $K^{\prime} \subset Z$ such that $\zeta C=C^{\prime}$. The homeomorphism $\zeta$ will be obtained as the composition of homeomorphisms $\zeta_{1}, \cdots, \zeta_{7}$ (i.e., $\zeta=\zeta_{7} \zeta_{6} \zeta_{5} \zeta_{4} \zeta_{3} \zeta_{2} \zeta_{1}$ ). We set $K_{0}=K, C_{0}=C$, and agree that whenever a homeomorphism $\zeta_{i}$ of $K_{i-1}$ has been defined, then $K_{i}=\zeta_{i} K_{i-1}$ and $C_{i}=\zeta_{i} C_{i-1}$. Let us now define the homeomorphisms $\zeta_{i}$. 
$\left(\zeta_{1}\right)$ The affine hull aff $K_{0}$ of $K_{0}$ is separable and thus admits a total sequence $f_{\alpha}$ of continuous affine functionals which are uniformly bounded on $K_{0}$ and such that $H \cap$ aff $K_{0}=\left\{p: f_{1} p=-1\right\}$ and $H^{\prime} \cap$ aff $K_{0}=\left\{p: f_{1} p=1\right\}$, where $H^{\prime}$ is $K_{0}$ 's other supporting hyperplane which is parallel to $H$. For each $p \in K_{0}$, let $\zeta_{1} p=\left(f_{1} p, f_{2} p / 2\right.$, $\left.\cdots, f_{n} p n, \cdots\right) \in l^{2}$. Then $\zeta_{1}$ is an affine homeomorphism of $K_{0}$ onto a compact convex subset $K_{1}$ of $l^{2}$, the set $C_{1}=\zeta_{1} C_{0}$ lies in the intersection of $K_{1}$ with its supporting hyperplane $J_{-1}=\left\{x \in l^{2}: x^{1}=-1\right\}$, and $K_{1}$ is supported also by the hyperplane $J_{1}=\left\{x \in l^{2}: x^{1}=1\right\}$.

$\left(\zeta_{2}\right)$ Let $v_{-1} \in C_{1} \subset K_{1} \cap J_{-1}$ and $v_{1} \in K_{1} \cap J_{1}$. For each $x \in l^{2}$ let $\pi x=x-x^{1} \delta_{1}=\left(0, x^{2}, x^{3}, \cdots\right)$. Then define $\zeta_{2}$ as follows :

$$
\begin{aligned}
\left(\zeta_{2} x\right)^{1} & =x^{1} \\
\pi\left(\zeta_{2} x\right) & =\pi x+\frac{1}{2} x^{1}\left(\pi v_{-1}-\pi v_{1}\right)-\frac{1}{2}\left(\pi v_{1}+\pi v_{-1}\right) .
\end{aligned}
$$

Then $\zeta_{2}$ is an affine homeomorphism, $\zeta_{2} v_{-1}=-\delta_{1}$, and $\zeta_{2} v_{1}=\delta_{1}$. The set $K_{2}$ is supported by the hyperplanes $J_{-1}$ and $J_{1},-\delta_{1} \in C_{2} \subset K_{2} \cap J_{-1}$, and $\delta_{1} \in K_{2} \cap J_{1}$.

$\left(\zeta_{3}\right)$ Let $B^{\prime}$ be a spherical cell in $l^{2}$ which is tangent to the hyperplane $J_{1}$ at the point $\delta_{1}$ and such that $C_{2} \subset B^{\prime}$. Let $B$ denote the set of all $x \in B^{\prime}$ for which $x^{1}+1$ is no less than the distance from $\pi x$ to the set $\pi C_{2}$. Then $B \cap J_{-1}=C_{2}, B \cap J_{1}=\left\{\delta_{1}\right\}$, and $B$ is a bounded closed convex set. Let $\mu$ denote the gauge functional of $B$, and for each $x \in K_{2}$ let $\zeta_{3} x=(1+\mu x)^{-1} x$. Since $0 \in K_{2}$, it follows from II that $\zeta_{3}$ is a homeomorphism and the set $K_{3}$ is convex. Clearly $K_{3}$ is supported by the hyperplanes $J_{-1 / 2}$ and $J_{1 / 2} ; K_{3} \cap J_{-1 / 2}=C_{3}$ and $K_{3} \cap J_{1 / 2}=\left\{\delta_{1} / 2\right\}$.

$\left(\zeta_{4}\right)$ By well-known properties of Hilbert spaces, there exists an affine isometry $\zeta_{4}$ of $l^{2}$ onto $Z$ such that $\zeta_{4}\left(\delta_{1} / 2\right)=(0,1)$ and $\zeta_{4} J_{-1 / 2}$ $=X_{0}$. Then of course $K_{4} \subset Y \cup X_{0}$ and $C_{4} \subset X_{0}$. From $I$ it follows that the set $K_{4} \cap X_{1 / 2}$ admits a nonsupport point $z$ relative to $X_{1 / 2}$ and the set $C_{4}$ admits a nonsupport point $z_{0}$ relative to the flat $V=\mathrm{cl}$ aff $C_{4}$, the smallest closed flat containing $C_{4}$. For $r \in[0,1]$, let $z_{r}$ be defined as follows:

$$
z_{r}= \begin{cases}(1-2 r) z_{0}+2 r z & \text { for } r \in[0,1 / 2] \\ (2-2 r) z+(2 r-1)(0,1) & \text { for } r \in[1 / 2,1] .\end{cases}
$$

Then for each $r \in] 0,1\left[, z_{r}\right.$ is a nonsupport point of the set $K_{4} \cap X_{r}$ relative to $X_{4}$.

$\left(\zeta_{5}\right)$ Let $h$ be as in III. Then $\mathrm{cl} h V$ is a flat of infinite dimension and infinite deficiency in $X_{0}$, so (again by well-known properties of 
Hilbert spaces) there is a linear isometry $\eta$ of $Z$ onto $Z$ which carries $Y$ onto $Y, X_{0}$ onto $X_{0}$, and $\operatorname{cl} h V$ onto $W$. For each $x \in K_{4}$, let $\zeta_{5} x$ $=h^{-1} \eta h x$. Then $\zeta_{5}$ is a homeomorphism. Although the set $K_{5}=\zeta_{5} K_{4}$ may fail to be convex, the following assertions can be verified:

(i) for each $r \in[0,1]$, the set $D_{r}=K_{5} \cap X_{r}$ is compact and convex;

(ii) $D_{0}=C_{5}$ and $D_{1}=\{(0,1)\}$;

(iii) for each $r \in\left[0,1\left[\right.\right.$, cl aff $D_{r}=X_{r}$;

(iv) the function $D_{r} \mid r \in[0,1]$ is continuous, where $\left\{D_{r}: r \in[0,1]\right\}$ is metrized by means of the Hausdorff metric;

(v) with $q_{r}=\zeta_{5} z_{r}, q_{r}$ is a nonsupport point of the set $D_{r}$ (relative to $\left.X_{r}\right)$ for each $r \in[0,1[$;

(vi) the function $q_{r} \mid r \in[0,1]$ is continuous.

$\left(\zeta_{6}\right)$ For each $(x, r) \in K_{5}$ let

$$
\zeta_{6}(x, r)=\left(q_{r}+\left(1+\left\|x-q_{r}\right\|\right)^{-1}\left(x-q_{r}\right), r\right) .
$$

From II and (v) above it follows that for each $r \in[0,1[$, the set $G_{r}=\zeta_{6} D_{r}$ is elliptically convex relative to $X_{r}$. And it is evident that conditions (i)-(vi) above remain satisfied when $D_{r}$ is replaced by $G_{r}$ in (i) $-(\mathrm{v}), K_{5}$ by $K_{6}$ in (i), and $C_{5}$ by $C_{6}$ in (ii).

$\left(\zeta_{7}\right)$ Now recall the definition of the set $K^{\prime}$, and for each $r \in[0,1]$, let $K_{r}^{\prime}=K^{\prime} \cap X_{r}$. Then $K_{1}^{\prime}=\{(0,1)\}=G_{1}$, and for each $r \in[0,1$ [ the sets $G_{r}$ and $K_{r}^{\prime}$ are both compact elliptically convex subsets of $X_{r}$. Let $\zeta_{7}(0,1)=(0,1)$, and for each $r \in\left[0,1\right.$ [ let $\zeta_{7}$ be the Kellerhomeomorphism of $G_{t}$ onto $K_{r}^{\prime}$ (as discussed in $[1 ; 2 ; 3 ; 4]$ ). Then clearly $\zeta_{7} K_{6}=K^{\prime}$ and $\zeta_{7} C_{6}=C^{\prime}$. That $\zeta_{7}$ is a homeomorphism follows from condition ( $v$ ) above in conjunction with the continuity property of the Keller-homeomorphisms expressed in 4.2 of [3]. The proof is complete.

\section{REFERENCES}

1. Ott-Heinrich Keller, Die Homoiomorphie der kompakten Mengen im Hilbertschen Raum, Math. Ann. vol. 105 (1931) pp. 748-758.

2. Victor Klee, Convex bodies and periodic homeomorphisms in Hilbert space, Trans. Amer. Math. Soc. vol. 74 (1953) pp. 10-43.

3. - Some topological properties of convex sets, Trans. Amer. Math. Soc. vol. 78 (1955) pp. $30-45$.

4. - Homogeneity of infinite-dimensional parallelotopes, Ann. of Math. vol. 66 (1957) pp. $454-460$.

UNIVERSITY OF WASHINGTON 